\title{
Additive (nano)manufacturing perspectives: the use of nanofillers and tailored materials
}

\author{
Elias P. Koumoulos ${ }^{1,2, *}$, Eleni Gkartzou ${ }^{1}$, and Costas A. Charitidis ${ }^{1}$ \\ ${ }^{1}$ RNanolab, National Technical University of Athens, School of Chemical Engineering, 9 Heroon Polytechniou St., Zographos, \\ Athens 15780, Greece \\ 2 BioG3D- New 3D Printing Technologies, Technological Cultural Park Lavrion, Athens, Greece
}

Received: 16 March 2017 / Accepted: 15 October 2017

\begin{abstract}
Additive manufacturing (AM) is identified to cost-effectively lower manufacturing inputs and outputs in small batch production, widely employed in customized and high-value manufacturing chains, such as aerospace and medical component manufacturing. Additive manufacturing has the potential to significantly lower life cycle energy demands of products and their $\mathrm{CO}_{2}$ emissions. Moreover, AM holds promise of overturning many aspects of the economics of manufacturing, as it pays no heed to unit labour costs or traditional economies of scale. Advances in AM technology are yielding faster production times and enabling objects to be printed in multiple combinations of materials, colours and surface finishes. A significant portion of these advances lie on the development of advanced materials for AM processes, which is undeniably one of the main driving forces of the transition from Rapid Prototyping to the Direct Digital Manufacturing era. Industries are nowadays at the inflection point for AM technologies, which have moved from a much-hyped but largely unproven manufacturing processes, to a mature technological solution, with numerous competitive advantages and the ability to produce real, innovative, complex and robust products.
\end{abstract}

Keywords: additive manufacturing / nanomaterials / 3D nanofabrication-printing / microfluidic devices / manufacturing upscaling

\section{Introduction}

Additive manufacturing (AM) was introduced around the mid 80 s and according to the prevailing view is currently at the starting point of triggering a new industrial revolution. The recent spread of $3 \mathrm{D}$ printers is the result of the expiration of the patent for the FDM (Fused Deposition Modelling) in 2009. Respectively, at an industrial scale, similar spread has been accomplished from 2014, when the patent for the SLS (Selective Laser Sintering) technology expired.

$\mathrm{AM}$ is identified to cost-effectively lower manufacturing inputs and outputs in small batch production, widely employed in customized and high-value manufacturing chains, such as aerospace and medical component manufacturing. This method lowers energy use, resource demands and related $\mathrm{CO}_{2}$ emissions over the entire product life cycle, induces changes in labour structures and generates shifts towards a digitalized and localized supply chain. In their case study, Gebler et al. (2014) created a model showing that AM has the potential to reduce costs by $170-593$ billion US $\$$, total primary energy supply by $2.54-9.30 \mathrm{EJ}$ and $\mathrm{CO}_{2}$

\footnotetext{
* e-mail: elikoum@chemeng.ntua.gr
}

emissions by $130.5-525.5 \mathrm{Mt}$ by 2025 . The great range within the saving potentials is a result of uncertainties of predicting market and technology developments [1].

AM has the potential to significantly lower life cycle energy demands of goods and their $\mathrm{CO}_{2}$ emissions. Reeves et al. has demonstrated in a case study of a structural airplane component that manufacturing-related energy demands and $\mathrm{CO}_{2}$ emissions can be lowered by up to $75 \%$ if AM processes are employed. The AM-induced lightweight design further adds usage savings which amount to $63 \%$ savings in energy and $\mathrm{CO}_{2}$ emissions over the entire life cycle of the product. This shows that AM has a great environmental potential not simply limited to aspects of the manufacturing process [1]. Various application fields and respective specific AM processes are outlined in Table 1 . One of the most widely used by the industry AM technologies is SLS technology, to address the needs for high-performance applications and manufacturing of intricate components of high geometrical complexity. Systems employing SLS technology lay the material in powder form and use a high-power laser beam in order to fuse particles and form the successive layers of the object. This technique is compatible with a wide variety of plastic, metallic, ceramic, glass materials and any material that exists in a powdery form and can be fused. 
Table 1. Application fields and respective specific processes.

\begin{tabular}{ll}
\hline Application field & Specific process \\
\hline Car industry & Integration of many parts in a unified composite part \\
& Construction of manufacturing means \\
& Manufacturing of spare parts and accessories \\
& Fast standardization \\
& Manufacturing of components of complex geometry \\
Aerospace/aeronautics & Control of density, mechanical properties \\
& Manufacturing of light-weight components. \\
& Planning of surgical operation with the use of accurate \\
Medicine/pharmaceutical & anatomic models that are based on computed tomography (CT) \\
industry & or the magnetic resonance imaging (MRI) data \\
& Development of patient-specific orthopaedic implants and prosthetics \\
& Use of 3D printed corps simulations for medical training purposes (anatomy). \\
& Printing of biodegradable scaffolds for tests during the development \\
phase of the medicinal products & Manufacturing of accessories of complex geometry \\
Creation of adjusted protective equipment for better application and use & Creation of prototypes of multiple colours and composite \\
\end{tabular}

Through a layer-by-layer, digitally controlled printing process, AM technology offers high flexibility in fabricating 3D models and custom parts with structural and mechanical complexities that are unmatched by the traditional machining processes [2]. This technology is combined with selected materials and its spatial resolution is limited to around tens of micrometers by the inherent processes and mechanisms. As a challenge, fabricating 3D structures with nanoscale resolution and from a broad range of engineering materials is now emerging. In this respect, research activities focus on inventing new tools, methods, mechanisms and systems that are capable of processing materials at the nanoscale, aiming at the development of heterogeneous material architectures that significantly increase materials performance and broaden their functionality by nanostructuring and nanosystem integration.

Nowadays, the production and tailoring of advanced composites attracts economic and environmental interests and efforts are made on the manipulation and processing of these materials in order to enhance their performance. Among their competitive advantages are their ability to form light-weight structures with enhanced mechanical properties, along with a variety of integrated "smart properties", which translate into their capacity to be selfcleaning, self-healing, with memory, and have tailored anticorrosion and wear resistance properties. Such materials can generate and store energy from renewable sources [3-6], with a low production cost. Moreover, the replacement of conventional materials (e.g. steel or aluminum alloys), with advanced composite materials (usually prepared in a polymer matrix), can lead to the reduction of the environmental impacts of the final structures, in the form of fuel savings achieved due to the efficient operation of lightweight vehicles or the application of eco-friendly processes with a lower installation cost [7]. However, it is essential to balance these life-cycle energy advantages with possible highly energy-intensive advanced composites production [8].

In order to perform a holistic assessment of the properties of advanced composite materials, aspects regarding both energy savings as well as environmental impacts [9] are two considerations which should be taken into account during both phases (production and use) of their composition. That is, without neglecting their end-oflife and material substances, which can be inorganic, organic or hybrid organic-inorganic materials [10-14].

\section{Manufacturing scale: through upscaling, from the lab to the fab and industrial scale}

AM will overturn many of the economics of manufacturing as it pays no heed to unit labour costs or traditional economies of scale. Designs can be quickly adjusted, so flexible manufacturing and mass customization can be achieved with minor capital investment. However, even if the crucial point of a lab's printer is that it combines size and low cost (printers used by hobbyists are not expensive, but they have small building volume), many would find it hard to make anything larger than a coffee cup. Those used by engineering companies are significantly more expensive -and even these systems might face difficulties with printing an object at the size of a small boat $[15,16]$. Additionally, the gradual replacement of the multiplicity of skills used by labourers on a building site and decision making with a $3 \mathrm{D}$ printer is still at an initial stage and will take some time to be realised.

AM is not intended for replacing mass manufacturing. Note that the finish and durability of some printed items can still fall short of producers demands and nor can 3D printers crank out zillions of identical parts at low cost, as 

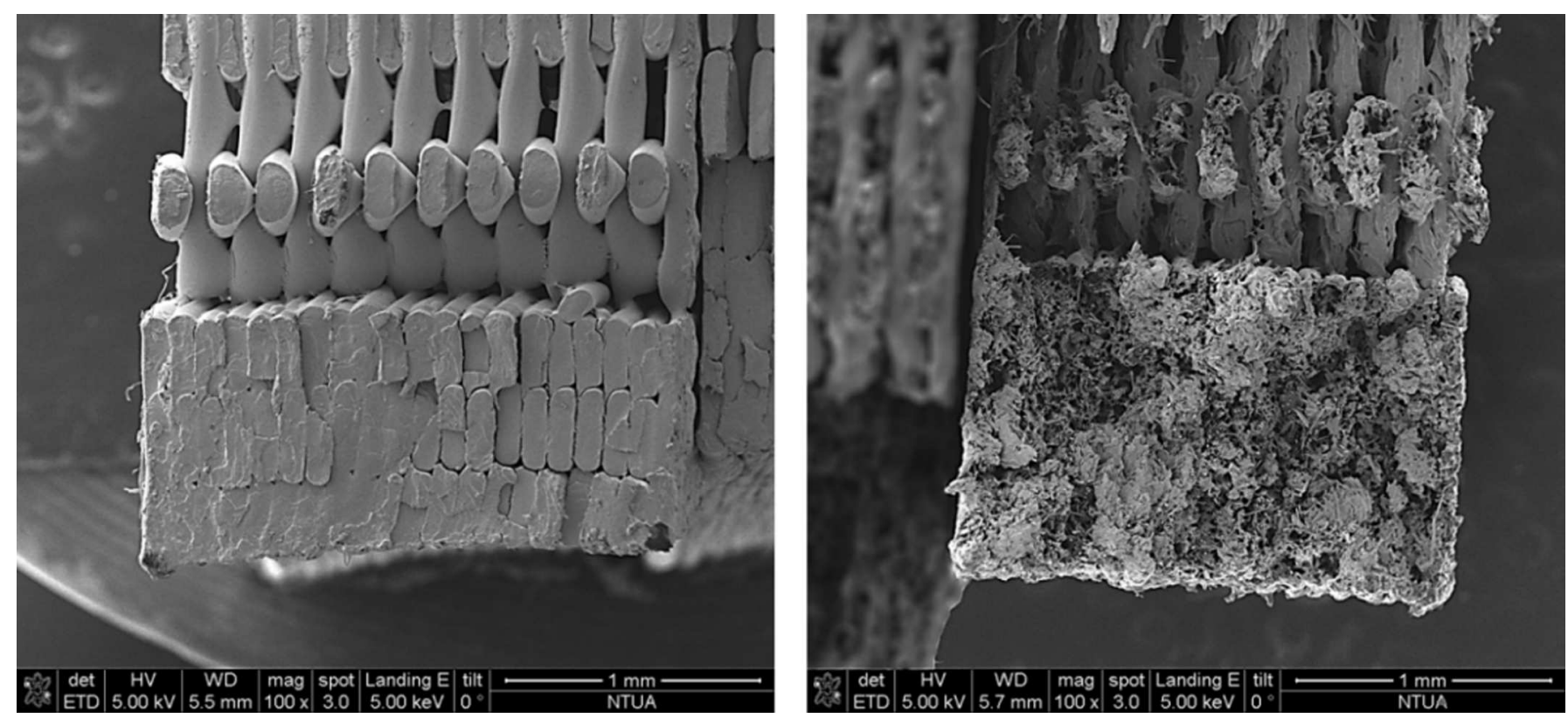

Fig. 1. Surface morphology of fractured tensile specimens from pure polymer (left) and carbon fiber reinforced polymer (right) produced by FDM. The composite material exhibits complex fracture mechanisms, arising from the geometrical characteristics of the $3 \mathrm{D}$ printed structure, processing conditions and the interactions between the reinforcing fibers and polymer matrix.

mass-manufacturing lines can. Nevertheless, 3D printers have their unique advantages, which is why they are employed in various manufacturing applications by some of the world's biggest manufacturers, such as Airbus, Boeing, GE, Ford and Siemens [17]. Industrial AM is currently at a turning-point, since more and more industrial units use these techniques to produce customised tools and small components. The commencement of this new industrial "revolution" is confirmed in a survey conducted in 2014, in which more than 100 construction companies participated. The research showed that $11 \%$ of the companies had already substituted traditional manufacturing methods by AM for mass manufacturing of individual parts or integrated products. Three years after the aforementioned research was carried out, one can safely assume that this percentage has certainly sprung. Indeed, according to a new PwC survey conducted at the end of 2015 , it is estimated that $42 \%$ of large industries in Northern America will be using AM and specifically SLS manufacturing for a large portion of their operations by 2020. Further enhancement of AM technology uptake will be achieved, as the range of compatible material options continues to expand, to address application-specific needs. AM has begun to integrate advanced materials that appeal to the designers for high performance applications, and reflect the recent advances in material science for ceramic, cement, glass, metallic materials and alloys as well as polymer (nano)composites reinforced with carbon nanotubes and fibers. However, the integration of new types of materials also requires new sets of standards and optimisation of the AM process, as well as advanced characterisation methods and in-line monitoring, as their final properties exhibit high variability and significant dependence on processing conditions and structural characteristics. For example, surface morphology of fractured tensile specimens from pure polymer and carbon fiber reinforced polymer produced by FDM are presented in Figure 1. In the case of the composite material, highly complex fracture mechanisms can be observed, arising from interrelated factors (geometrical characteristics of the 3D printed structure, processing conditions and the interactions between the reinforcing fibers and polymer matrix), which need to be optimized to increase the robustness of the AM process and meet industrial requirements. Despite the fact that the direct costs of manufacturing products with these new methods and materials are usually higher, the flexibility offered by AM technologies implies that the total cost can be substantially lower (Tab. 2)

\section{3D Nanofabrication-printing of nanomaterials}

AM has provided a pathway for low cost and flexible manufacturing of personalized components and one-off parts; however, such techniques are less ubiquitous at the nanoscale [18], where nanofabrication is dominated by lithography tools that are of rather high cost for small- and medium-sized enterprises. Adding nanomaterials such as carbon nanotubes (CNTs), nanowires (NWs), and quantum dots (QDs) to host matrices such as polymers, metals, and ceramics via AM has the potential to enable greater capabilities in (nano)composites manufacturing. Incorporating (nano)materials to $\mathrm{AM}$ is realized via two ways; either use of AM for host matrix material with intermittent stoppages of the batch print job and introduction of nanomaterials automatically or manually, or pre-mixing of the nanomaterials into the host matrix, followed by AM of the nanocomposite mixture as a complete part. This will lead to enhancement of mechanical properties, increase of 


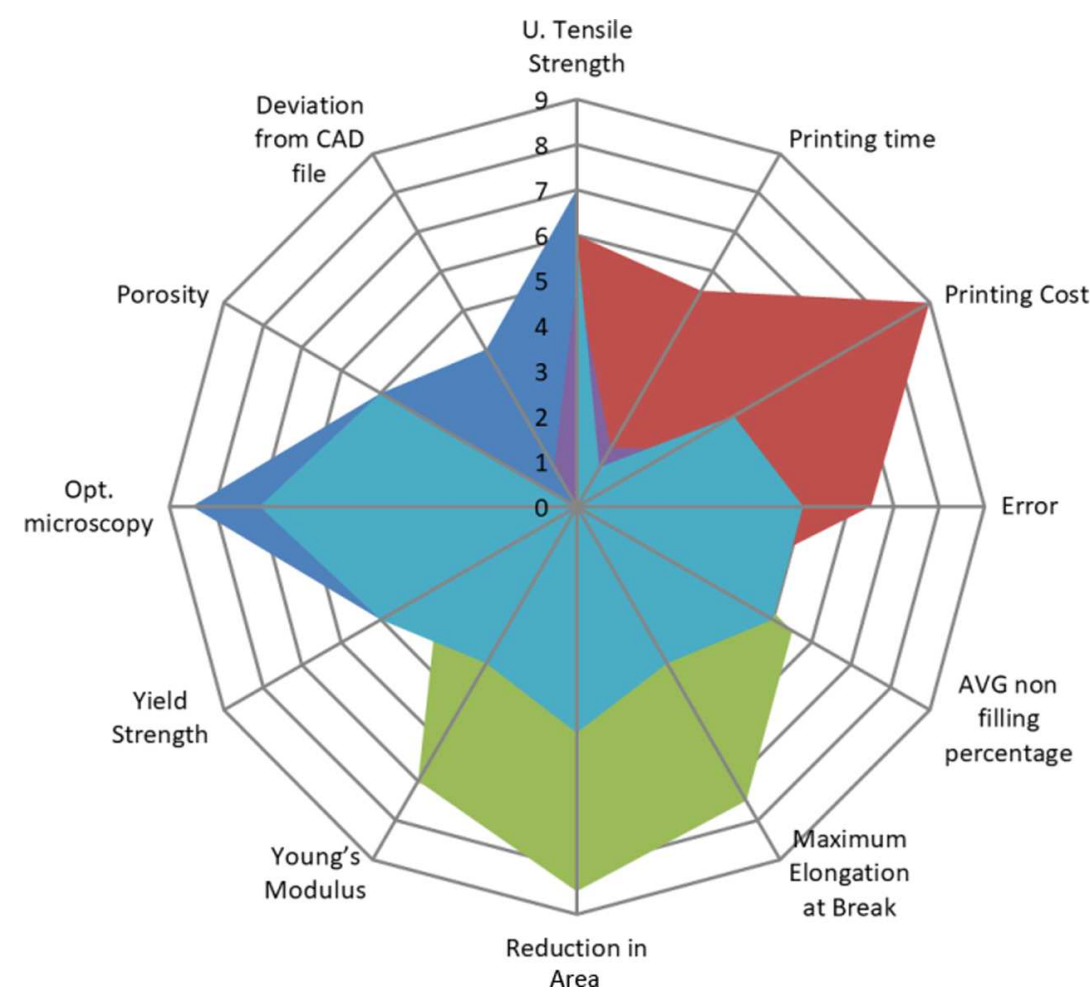
- PLA 100\% infill, 0 degrees printing angle
a PLA 100\% infill, 45 degrees printing angle - PLA 100\% infill, 75 degrees printing angle - CarbonFil $100 \%$ infill, 0 degrees printing angle a Carbonfil $100 \%$ infill, 45 degrees printing angle

Fig. 2. Radar chart providing comparison of i) polylactic acid (PLA) and ii) Polyethylene terephthalate glycol-modified (PETG) with carbon filler (CarbonFil ${ }^{\mathrm{TM}}$ ), with a scale factor from 0 to 10 (with 10 being the most significant impact).

Table 2. Advantages and unresolved issues of AM processes.

Complex geometries.

Variety of products, due to the convenient readjustment

Less waste

Zero restrictions in the product design

Easy handling
Long printing time.

High initial manufacturing cost

Non-consecutive

Manufacturing process

Mechanical properties thermal and electrical conductivity, lower sintering temperatures, and will affect dimensional accuracy, as the incorporation of carbonaceous and metallic nanoparticles has been found to decrease deflection and shrinkage in various AM processes, due to more effective heat dissipation and lower temperature processing [19]. However, AM methods have their own limitations when nanoparticles are introduced, depending on the principle of operation, such as nozzle clogging for FFF, aggregation within liquid media and viscosity increase for ink-jet and stereolithography, nano-powder aggregation and decreased flowability in SLS processes, as well as rough surface finish of printed parts, etc. [20]. A radar chart providing comparison of (i) polylactic acid (PLA) and (ii) polyethylene terephthalate glycol-modified (PETG) with carbon filler (CarbonFil ${ }^{\mathrm{TM}}$ ) is provided in Figure 2 with a scale factor from 0 to 10 (with 10 being the most significant impact). In Figure 3, two types of void defects at different scales in polymer matrices are depicted, introduced by the FDM process and nanoparticle agglomeration respectively. These voids can have a significant impact on crack formation and overall performance of 3D printed nanocomposites, therefore nanoparticle homogeneity in host matrices and tailored processing profiles are vital prerequisites for realizing their full potential.

\subsection{Nanoparticles and nanostructures in AM processes}

Stabilized nanoparticles in a host matrix can be employed to achieve a desired distribution of nanoparticles in the matrix and thus to protect them from agglomeration, oxidation, and corrosion. For example, laser-assisted AM techniques can be used to prepare porous core-shell polymer structures, containing different encapsulated nanoparticles distributed heterogeneously over the sintered polymer. In literature, functionally graded 3D parts with the structural ordering of iron oxide particles and the respective optimal laser-processing regimes are reported [21], aiming at potential medical applications for the tissue engineering scaffolds and cell targeting systems. This approach renders possible the manipulation of fundamental material properties in objects (through nanomaterials) 

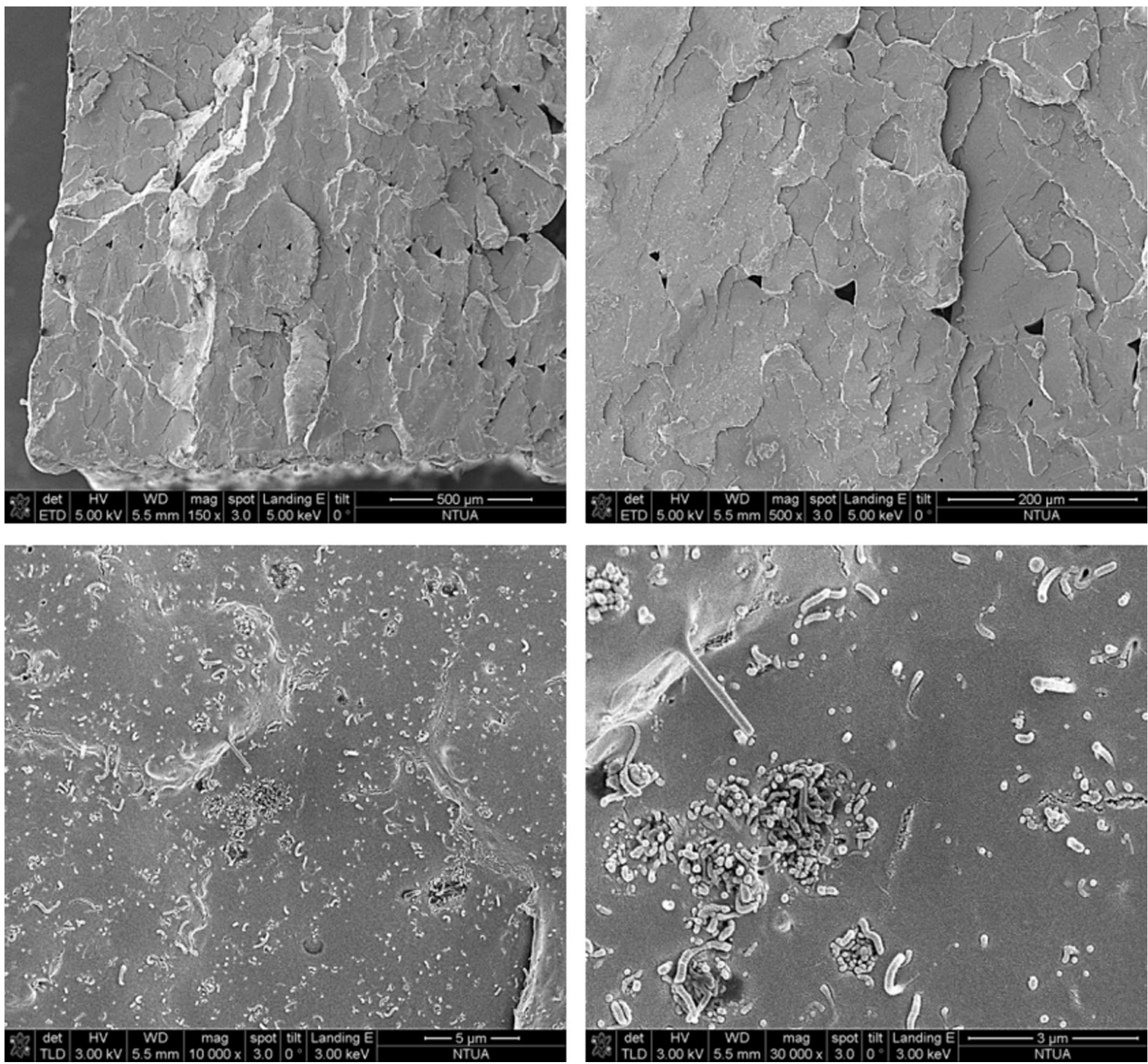

Fig. 3. Types of void defects at different scales in polymer matrices, introduced by the FDM process (top left, right) and nanoparticle agglomeration (bottom left, right) respectively.

and achieve customized geometries, reduced time between design iterations, single tool manufacturing, and increased parts integration (through AM) [22]. For example, during the last decade significant progress has been made in the field of resonant optics; for this, 3D direct-write synthesis approach is reported [23]. Direct-write fabrication of 3D metallic nanoarchitectures with spatial nanometer resolution via electron-stimulated reactions, which is applicable on virtually any material and surface morphology, paves the way for a new generation of 3D nano-plasmonic architectures that can be printed on-demand. Nonetheless, the compatibility of the materials developed and AM techniques is of major importance [24]. Materials with a high level of flowability are required in most selective deposition AM processes, while nanofillers such as nano-biomaterials, carbon based nanomaterials, nanoclays and metallic nanoparticles, along with the respective host matrices (e.g. thermoplastics and thermosets) can be adapted for AM processing of multifunctional materials. From a fabrication point of view, high-precision automated machinery and equipment or moving stages are another key factor for further increasing the resolution of structures produced.

\subsection{Nanomaterials-based bio-inks}

In scaffold application field, AM technologies have great advantages over traditional fabrication methods in terms of controlling the porosity, pore size, and interconnectivity 


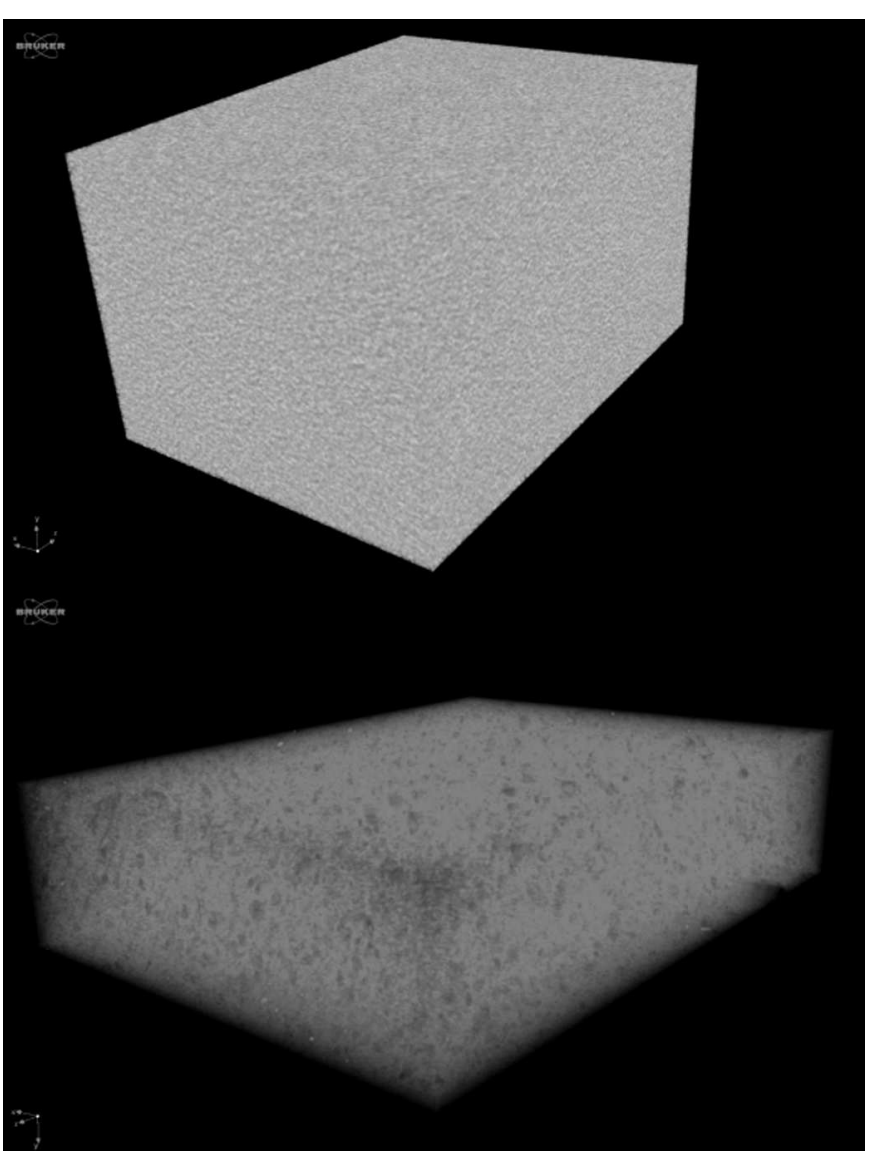

Fig. 4. Micro-computed tomography images of polymer (top) and polymer-CNT $10 \%(\mathrm{w} / \mathrm{w})$ (bottom) samples.

of the porous network. These methods can fabricate 3D scaffolds with high precision, thus can be used to standardize 3D scaffolds [25]. Among various fabrication methods, two-photon laser-based nanofabrication and controlled electrospinning are widely reported in various areas of tissue engineering due to their ability to fabricate structures with high surface to volume ratio and highly interconnected porous architecture at submicrometer resolution. The use of these methods results in precise $3 \mathrm{D}$ scaffolds mimicking the organization and structure of the extracellular matrix. The challenges induced in future development of 3D nanoscaffolds include the increase of the precision of scaffold fabrication systems, the identification new biomaterials, and incorporation of biomolecules to trigger specific cell behaviours such as adhesion, proliferation, and differentiation [26]. Additionally, the need to have mixed cell populations, biomimetic material properties, and chemical gradients has led to exploration of nanomaterials-based bioinks. Novel nanomaterials are in demand for cells and tissues to promote tissue regrowth and regeneration. Specifically, with the vision of functional 3D bioprinted organ successful implantation into a live human being, a clinical potential is opened, with further research in various advanced AM modalities. Bioplotting is one of the most promising technologies for the development of a fully functioning, multicellular organ that can integrate into and thrive within a human body in the future [27].

\subsection{Carbonaceous nanomaterials in AM processes}

Carbon nanotubes are known for their mechanical, electrical, and thermal properties, which initially makes them a suitable candidate for integration in AM processing of polymers. Nanotubes can be used as carriers of chemical or biologically active materials in advanced (bio)sensing applications (proteins, poisonous gases, fuel components, and even melted metals), while their increased affinity to lipid structures enables them to form stable complexes with peptides and DNA-oligonucleotides and even encapsulate these molecules, which is useful for targeted drug delivery and numerous medical applications. However, their homogeneous dispersion in polymeric solutions and materials is crucial towards enhancement of CNT-based AM materials. For example, in case of FDM processing of thermoplastic nanocomposites, aggregation of CNTs is frequently reported and can be detrimental to FDM, possibly causing blockages at the nozzle and flux instability while printing (clogging). This is confronted by tailoring the concentration of CNTs which should preferably not surpass the percolation threshold, while maintaining key properties for AM process (mechanical integrity, flux, rheology, etc.) [28]. In Figure 4, micro-computed tomography images of polymer (left) and polymer-CNT 10\% w/w (right) samples are presented (agglomeration is evidenced for the case of nanocomposite).

Fabrication of highly conductive CNTs/PLA nanocomposites used as 3D printable conductive inks for fabrication of conductive scaffold structures is reported as applicable in liquid sensors [28]. The structural parameters of liquid sensors are controlled through AM, while their influence on the sensitivity of the obtained sensor can be useful for structures made from repeated patterns of filaments, such as for sensors in form of textiles [29]. Carbonaceous nanomaterials (such as CNTs, graphene, carbon black) have also been explored as additives for SLS; distribution of the nanoparticles remains an important issue to be solved, however simple mixing techniques (initial dispersion of MWCNT powder in solvents, suspension through magnetic stirring, washing etc.) are often used to obtain homogeneity.

\section{The case of $A(N) M$ for $3 D$ microfluidic devices}

In the field of microfluidic applications, AM has begun to embrace the range of sizes and materials that appeal to the developers of microfluidic devices and reflect the recent advances in polymer-based systems, where no etching or dissolution processing is required and are thus more environmentally friendly and economically efficient [30]. Commercially available 3D printing systems for microfluidic devices fabrication and various 3D printed polymeric microfluidic components (microvalves and micropumps, droplet and emulsion chips, micromixers, microreactors, sensor cartridges and medical devices etc.) have already emerged in the market [31]. In the case of (bio) chemical sensors fabrication through AM, novel materials should be further be developed with specific functionalities 
and improved processability characteristics for the respective AM technology that will be employed, in order to present the necessary detection capabilities. The simultaneous processing and integration of materials with different AM technologies, properties and functionalities in a single manufacturing process will render the formation of new selective biorecognition systems on polymer surfaces possible, with high versatility and flexibility in incorporating novel bio-probes in ink formulations, thus meeting the demands of a more and more dynamic market, while maintaining cost efficacy and sustainability. The above technologies can be autonomously applied in different applications, e.g. 3D electronics with carbon-based conductive inks for medical devices and sensing applications.

\subsection{Microfluidic devices on polymer substrates}

Polymers have recently found a variety of applications in microfluidic systems, due to the wide range of low-cost polymeric materials available on the market, their ease of recyclability and their highly adjustable physicochemical characteristics, which can be tailored to address the specific needs of different applications. In this context, an important area of applied research concerns the technology transfer from the first-generation microfluidic devices based on silicon or glass to polymeric matrices. Polymer microsystems in materials such as polymethylmethacrylate (PMMA), polycarbonate (PC), polystyrene (PS), polyethyleneterephthalate (PET), Cyclic Olefin Copolymer (COC) or poly(dimethylsiloxane) (PDMS) microstructures can be produced in high volumes with scalable processing technologies, however the increasing awareness of limited resources, have motivated research and optimisation of renewable alternatives to traditional petroleumderived plastics, such as bio-based materials that are sourced from carbon-neutral feedstocks [32].

Poly lactic acid (PLA) is a widely studied commercial bio-based/biodegradable thermoplastic polyester, which is derived from renewable resources such as cornstarch and sugarcane. The carbon in PLA originates from atmospheric carbon dioxide, which is immobilized in glucose by photosynthesis; therefore, its carbon footprint during production and disposal is low, compared to other petrobased polymers. Thermoplastic filaments based on PLA are dominant materials in FDM applications, due the highstrength, high-modulus, biocompatibility, rheological properties in molten state, low toxic emissions and crystallisation kinetics of PLA, which enable fast melting, extruding and selectively depositing of fibers for the formation of a predefined shape. However, PLA suffers from a series of drawbacks like brittleness, low heat deflection temperature, poor processability, and low melt strength [33]. Therefore, blending PLA with other polymers with desirable properties, along with the addition of environmentally friendly plasticizers and various strategies of compatibilization, is one of the most costefficient approaches to overcome these drawbacks, while maintaining its biodegradability and easy of recyclability. Furthermore, melt-blending of PLA-based materials with nanoparticles such as graphene, CNTs and organically- modified nanoclays, among others, is a well-established route for the scaled-up production of advanced nanocomposites with functional properties and enhanced thermal stability [34].

An important benefit of the aforementioned polymeric materials is their ease of recyclability and wide variety of end-of-life options, in combination of low carbon footprint of biobased thermoplastics during production and disposal. Polymeric materials can be developed to locally improve selected physical properties, e.g. fast heat dissipation and/ or enhanced dielectric properties near electrical components, while maintaining optimum processability characteristics for AM processing. Standard methodologies and protocols must be developed to assess the printability of the produced filaments, including melt behaviour, surface finish, dimensional stability, layer adhesion and mechanical performance for various test specimens. The emerging novel material solutions and technologies include hybrid AM processes (e.g. a combination of FDM and ink-jet technologies) involving the co-deposition and compatibilization of conductive inks and high-performance polymers, functionally graded morphologies and conformal integration of customized materials, which have the potential to drive advanced manufacturing industry forward, not only in the field of biosensor microfluidic applications, but also in other key industrial sectors, where there is a high demand for new sets of sustainable performant materials.

\subsection{Functional micro-component fabrication via Ink- Jet printing}

Ink-jet printing technologies offer a wide flexibility in processing ink formulations, whose constituents can provide numerous functionalities in polymer-based substrates, such as microelectrode formation and bio-interactive components for biochemical analysis. Conductive inks offer the opportunity to create conductive paths in order to incorporate electrical components in microfluidic devices, as the step of drop resolution of ink-jet technologies has advanced from $500 \mu \mathrm{m}$ (30 dpi) to several micrometers (9000 dpi) [21]. The most promising solution to prepare conductive inks is the use of nanoparticle suspensions. These nanoinks offer significant advantages such as: faster drying and ease of dispersion in water. However, one of the problems to be tackled is the development of stable colloidal suspensions, which prevent nozzle clogging and nanoparticle aggregations during solidification. Carbon-based nanomaterials, like CNTs or graphene, are highly promising for the preparation of conductive inks. It has been recently presented that graphene inks, based on the use of few layer graphene can produce high-concentration graphene dispersions, which have been tested in micro-supercapacitors with resistances between $\mathrm{k} \Omega$ and $\mathrm{M} \Omega$. This technology provides an efficient and low-cost method to fabricate a variety of graphene electronic devices with good performance and is a promising alternative for future commercial applications in printed and flexible electronics. As mentioned, the introduction of nanoparticles in liquid media causes a significant increase in the viscosity of the media, thus rendering difficult the formation of small droplets during inkjet deposition. In 
practice, a selected combination of carriers, additives and carbon nanoparticles occur, so as to achieve inks with the expected properties in terms of conductivity. The use of nano-intermediates, pre-dispersion of nanoparticles in suitable carriers help to create the dispersion with the needed stability and viscosity. For the development of stable conductive inks based on graphene, the most important feature to control is the viscosity of the medium, to ensure that it is feasible to create small droplets with the developed AM system. The development of inks containing biointeractive components is challenging in terms of the development of a stable ink formulation which preserves the intended functionality of the bio-interactive content in the bulk state, when printed and are compatible with the inkjet technology. Additional challenges relate to potential damage resulting from sheer stresses introduced during the jetting process, sedimentation, conglomeration, adhesion to the inner surfaces of the print head/nozzle, crystallisation at the jet nozzle, and rheologies which are outside the operational range of the print head (e.g. viscosity, surface tension, non-Newtonian behaviour etc.). Furthermore, modification/adaption of the print head may be necessary (e.g. geometries, hydrophobic/hydrophilic coatings, materials used, etc.) to prevent wetting/adhesion to the print head nozzle and nozzle plate; humidity and temperature control may be necessary to preserve functionality; and potential sterilisation and cross-contamination issues need to be addressed.

\section{Conclusions and future perspectives}

AM is a market with enormous growth potential, which will be fulfilled once the main remaining barriers to up-take are addressed. Although industrial AM still faces many challenges, it is a matter of time until AM processes replace traditional manufacturing methods in small batch production, widely employed in customized and high-value manufacturing chains, such as aerospace and medical component manufacturing. Advances in these technologies are yielding faster production times and new sets of performant materials, enabling objects to be printed in multiple combinations of materials, colours and finishes. Additionally, nanofabrication technologies through AM will become important tools in tissue engineering in the next few years, rendering possible the fabrication of high resolution $3 \mathrm{D}$ biomimetic environments. The ability to design geometric features, tune material properties through nanomaterial integration, and incorporate drug delivery platforms through multi-material selection indicates a revolution in tissue engineering and pharmaceutical applications. Furthermore, the simultaneous processing and integration of materials with different AM technologies, properties and functionalities in a single manufacturing process will render possible the formation of new selective biorecognition and Micro Total Analysis Systems, with high versatility and flexibility in incorporating novel bio-probes in ink formulations, thus meeting the demands of a more and more dynamic market, while maintaining cost efficacy and sustainability. Additionally, novel real-time characterisation techniques should be developed, to provide feedback for material development, targeting at optimum processability characteristics for a wide range of processing conditions typically involved in hybrid manufacturing processes. This paves the way for robust quality assurance and quality control of material behaviour during and after the manufacturing process [35], facilitating the establishment of technical standards and acceptance from industrial users. Overall, the challenge is to design machines with multifunctional printing capability and to combine different AM techniques and advanced materials. Parallel advances on both nanomaterials and nanofabrication will lead to the development of miniaturized functional devices easily adjustable for a wide range of application fields.

\section{References}

1. M. Gebler, A.J.M. Schoot Uiterkamp, C. Visser, A global sustainability perspective on 3D printing technologies, Energy Policy 85 (2015) 511

2. R. D'Aveni, The 3-D printing revolution, Harv. Bus. Rev. (2015) 40-48

3. L. Fang, S. Chen, T. Fang, J. Fang, C. Lu, Z. Xu, Compos. Sci. Technol. 138 (2017) 106-116

4. G. Ji, P. Zhang, J. Nji, M. John, G. Li, Recent Advances in Smart Self-healing Polymers and Composites, edited by G. Li, H. Meng (2015) pp. 293-363, ISBN: 978-1-78242-280-8

5. M. Naebe K. Shirvanimoghaddam, Appl. Mater. Today 5 (2016) 223-245

6. E. Gkartzou, E.P. Koumoulos, C.A. Charitidis, Manuf. Rev. 4 (2017) 1

7. F.C. Campbell, Structural composite materials, ASM Int. 05287G (2010) 10-27

8. C. Baillie, Green composites, in: Polymer Composites and the Environment, CRC Press, Taylor \& Francis, 2005, ISBN 9780849325762

9. Y.S. Song, J.R. Youn, T.G. Gutowski, Compos. Appl. Sci. Manuf. 40 (8) (2009) 1257-1265

10. E.P. Koumoulos, D.A. Dragatogiannis, I.A. Kartsonakis, E. Karaxi, T. Kehagias, C.A. Charitidis, Int. J. Struct. Integr. 7 (2016) 671-689

11. E.P. Koumoulos, T. Parousis, A.F.A. Trompeta, I.A. Kartsonakis, C.A. Charitidis, Plast. Rubber Compos. 45 (2016) 106-117

12. E.P. Koumoulos, I.A. Kartsonakis, A. Bakolas, C.A. Charitidis, Manuf. Rev. 3 (2016) 20

13. E.P. Koumoulos, P. Jagadale, A. Lorenzi, A. Tagliaferro, C. A. Charitidis, Compos., Eng. 80 (2015) 27-36

14. E.P. Koumoulos, P. Jagdale, I.A. Kartsonakis, M. Giorcelli, A. Tagliaferro, C.A. Charitidis, Polym. Compos. 36 (2015) 1432-1446

15. Additive manufacturing: Print me a jet engine, The Economist, The Economist Newspaper Limited, 2012

16. 3D printing: A third-world dimension, The Economist, The Economist Newspaper Limited, 2012

17. 3D printing scales up, The Economist, The Economist Newspaper Limited, 2013

18. D.S. Engstrom, B. Porter, M. Pacios, H. Bhaskaran, Additive nanomanufacturing - a review, J. Mater. Res. 29 (2014) $1792-1816$

19. O. Ivanova, C. Williams, T. Campbell, Additive manufacturing $(\mathrm{AM})$ and nanotechnology: promises and challenges, Rapid Prototyp. J. 19 (2013) 353-364 
20. H.N. Chia, B.M. Wu, Recent advances in 3D printing of biomaterials. J. Biol. Eng. 9 (2015) 1-14

21. I. Volyanskii, I.V. Shishkovsky, Laser-assisted 3D printing of functional graded structures from polymer covered nanocomposites: a self-review, in: Igor Shishkovsky (Ed.), New Trends in 3D Printing, InTech, 2016

22. T.A. Campbell, O.S. Ivanova, 3D printing of multifunctional nanocomposites, Nanotoday 8 (2013) 119-120

23. R. Winkler, F.P. Schmidt, U. Haselmann, J.D. Fowlkes, B.B. Lewis, G. Kothleitner, P.D. Rack, H. Plank, Direct-write 3D nanoprinting of plasmonic structures, ACS Appl. Mater. Interfaces 9 (2017) 8233-8240

24. R.D. Farahani, M. Dubé, D. Therriault, Three-dimensional printing of multifunctional nanocomposites: manufacturing techniques and applications, Adv. Mater. 28 (2016) 5794-5821

25. J.W. Lee, 3D Nanoprinting technologies for tissue engineering applications, J. Nanomater. 2015 (2015) 1-14

26. J.W. Lee, 3D Nanoprinting technologies for tissue engineering applications, J. Nanomater. 2015 (2015) 14 Article ID 213521, doi:10.1155/2015/213521

27. M.C. O'Brien, B. Benjamin, F. Scott, L.G. Zhang, Tissue Eng. Rev. 21 (2014) 103-114
28. S.F.A. Acquah, B.E. Leonhardt, M.S. Nowotarski, J.M. Magi, K.A. Chambliss, T.E.S. Venzel, S.D. Delekar, L.A. AlHariri, Carbon nanotubes and graphene as additives in 3D printing, in: Mohamed Berber (Ed.), Carbon NanotubesCurrent Progress of their Polymer Composites, InTech, 2016

29. K. Chizari, M. Daoud, A.R. Ravindran, D. Therriault, 3D Printing of highly conductive nanocomposites for the functional optimization of liquid sensors, Small 12 (2016) 6076-6082

30. A.K. Au, W. Huynh, L.F. Horowitz, A. Folch, 3D-printed microfluidics, Angew. Chem. Int. Ed. 55 (2016) 3862-3881

31. K.N. Ren, J. Zhou, H. Wu, Materials for microfluidic chip fabrication, Acc. Chem. Res. 46 (2013) 2396-2406

32. http://www.dolomite-microfluidics.com/webshop/microflui dic-chips-c-17

33. M. Nofara, M.C. Heuzey, P.J. Carreaub, M.R. Kamal, J. Randall. J. Rheol. 60 (2016) 637

34. K. Prashantha, F. Roger. J. Macromol. Sci. 54 (2016) 24-29

35. S. Tofail, E.P. Koumoulos, A. Bandyopadhyay, S. Bose, L. Donoghue, C. Charitidis, Mater. Today, in press (2017), https://doi.org/10.1016/j.mattod.2017.07.001

Cite this article as: Elias P. Koumoulos, Eleni Gkartzou, Costas A. Charitidis, Additive (nano)manufacturing perspectives: the use of nanofillers and tailored materials, Manufacturing Rev. 4, 12 (2017) 